\title{
In vitro PHYTOTHERAPY OF VECTOR SNAILS BY BINARY COMBINATIONS OF LARVICIDAL ACTIVE COMPONENTS IN EFFECTIVE CONTROL OF FASCIOLIASIS
}

\author{
Kumari SUNITA, Pradeep KUMAR, Vinay Kumar SINGH \& Dinesh Kumar SINGH
}

\begin{abstract}
SUMMARY
A food-borne trematode infection fascioliasis is one among common public health problems worldwide. It caused a great economic loss for the human race. Control of snail population below a certain threshold level is one of the important methods in the campaign to reduce the incidence of fascioliasis. The life cycle of the parasite can be interrupted by killing the snail or Fasciola larva redia and cercaria inside of the snail Lymnaea acuminata. In vitro toxicity of different binary combinations (1:1 ratio) of plant-derived larvicidal active components such as citral, ferulic acid, umbelliferone, azadirachtin and allicin against Fasciola redia and cercaria were tested. The mortality of larvae was observed at $2 \mathrm{~h}, 4 \mathrm{~h}, 6 \mathrm{~h}$ and $8 \mathrm{~h}$ of treatment. In in vitro condition azadirachtin + allicin $(1: 1$ ratio) was highly toxic against redia and cercaria $\left(8 \mathrm{~h} \mathrm{LC}_{50} 0.006\right.$ and $\left.0.005 \mathrm{mg} / \mathrm{L}\right)$. Toxicity of citral + ferulic acid was lowest against redia and cercaria larvae.
\end{abstract}

KEYWORDS: Lymnaea acuminata; Fascioliasis; Redia; Cercaria; Active larvicides.

\section{INTRODUCTION}

Fasciola spp is the causative agent of endemic fascioliasis in different parts of the world ${ }^{16}$. F. hepatica has worldwide distribution but predominates in temperate zones, while $F$. gigantica is found primarily in tropical regions ${ }^{2,14,15}$. This disease belongs to the plant-borne trematode zoonoses. The definite host is very broad and includes many herbivorous mammals, including humans. Bovine fascioliasis is very common in the eastern region of Uttar Pradesh, India ${ }^{22}$. SINGH \& AGARWAL ${ }^{25}$ reported that $94 \%$ of buffaloes slaughtered in local slaughtered house in Gorakhpur district are infected with $F$. gigantica. In northern India Lymnaea acuminata is the intermediate host of $F$. gigantica ${ }^{2}$. Although control of snail population below a threshold level is one of the important methods for effective control of fascioliasis ${ }^{9,11,13}$, yet snails are one of the important components in the aquatic ecosystem. Release of molluscicides in aquatic system for snail control also affects the other non-target organism. The Fasciola larval stages sporocyst, redia and cercaria are in division phases of $F$. gigantica in the snail body. If these larvae will be destroyed by plant molluscicides at sublethal concentration in the snail body, the rate of infection can be reduced without killing the snail. Binary combinations of different plants derived active larvicides such as citral (Zingiber officinale), ferulic acid, umbelliferone (Ferula asafoetida), azadirachtin (Azadirachta indica), and allicin (Allium sativum) ${ }^{30}$ were tested against Fasciola larvae in in vitro condition. It is a new approach to reduce incidence of the fascioliasis without killing the intermediate host snail.

\section{MATERIAL AND METHODS}

Animals: Infected adult Lymnaea acuminata $(2.6 \pm 0.20 \mathrm{~cm}$ in length) were collected locally. The snails were allowed to acclimatize for $24 \mathrm{~h}$ in laboratory condition. Each infected snail was dissected in a glass Petri dish containing $10 \mathrm{~mL} \mathrm{l}$ of dechlorinated water at $22{ }^{\circ} \mathrm{C}-24^{\circ} \mathrm{C}$. The $\mathrm{pH}$ of the water was 7.1-7.3 and dissolved oxygen, free carbon dioxide and bicarbonate alkalinity were $6.5-7.2 \mathrm{mg} / \mathrm{L}, 5.2-6.3 \mathrm{mg} / \mathrm{L}$ and 102.0-105.0 $\mathrm{mg} / \mathrm{L}$, respectively.

After dissection redia and cercaria were separated in different Petri dish containing $10 \mathrm{~mL} 1$ of dechlorinated water. These larvae were kept in dechlorinated tap water where they survived for up to $48 \mathrm{~h}$ in laboratory conditions. Snail L. acuminata and Fasciola gigantica were identified by Zoological Survey of India (ZSI), Kolkata.

Active larvicidal components: Citral, ferulic acid, umbelliferone, azadirachtin and diallyl disulfide were purchased from Sigma chemical Co. (U.S.A). Allicin was prepared by the method of SINGH \& $\mathrm{SINGH}^{27}$.

\section{Efficacy determination}

In vitro: In vitro efficacy of active larvicidal components in binary combinations (1:1 ratio) were performed in petri dish by the method of SUNITA \& $\mathrm{SINGH}^{30}$. Ten redia and cercaria of $F$. gigantica were separated into different Petri dishes containing $10 \mathrm{~mL} 1$ dechlorinated 


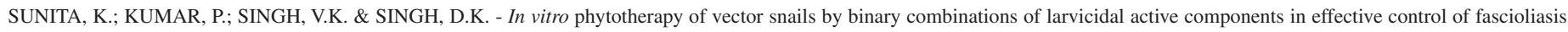
Rev. Inst. Med. Trop. Sao Paulo, 55(5): 303-8, 2013.

tap water. Mortality of redia and cercaria were observed after $2 \mathrm{~h}, 4 \mathrm{~h}, 6 \mathrm{~h}$ and $8 \mathrm{~h}$ of treatment. In control, no treatments were given in Petri dish. Usually, in in vitro conditions (control) survival of $72 \mathrm{~h}$ in dechlorinated water. Counting of larvae in control and treated groups was performed with the help of a microscope. Each experiment was replicated six times.

Effective concentrations to kill $50 \%\left(\mathrm{EC}_{50}\right)$, low and upper confidence limits (LCL and UCL), Slop-values, t-ratio, g value and heterogeneity factor were calculated with the help of the POLO computer program of ROBERTSON et al. ${ }^{19}$. One way ANOVA and product moment correlation coefficient was applied by the method of SOKAL \& ROHLF ${ }^{28}$.

\section{RESULTS}

In vitro larvicidal activity of different binary combinations (1:1 ratio) of active components against the redia and cercaria larva of $F$. gigantica was time and concentration dependent (Table 1 and 2). In in vitro treatments, binary combinations of azadirachtin + allicin were more efficient against redia and cercaria. The $8 \mathrm{~h} \mathrm{EC}_{50}$ of azadirachtin + allicin against redia/cercaria larvae in in vitro treatment was $0.006 \mathrm{mg} / \mathrm{L}$ $/ 0.005 \mathrm{mg} / \mathrm{L}$, respectively (Table 1 and 2). Citral + ferulic acid efficacy against both larval stages was lowest (Table 1 and 2). Significant $(p<$ 0.05 ) negative regression was observed in between exposure period and $\mathrm{EC}_{50}$ of different plant products. There was no mortality of $F$. gigantica larvae in control group.

The slope values were steep and separate estimation of LC based on each six replicate were found within the $95 \%$ confidence limit of $\mathrm{EC}_{50}$. The t-ratio was greater than 1.96 and the heterogeneity less than 1.0. The g-value was less than 0.5 at all probability levels (90, 95 and 99 respectively) (Table 1-2).

\section{DISCUSSION}

The present study clearly indicates that the active components of Zingiber officinale (citral), Ferula asafoetida (ferulic acid, umbelliferone), Azadirachta indica oil (azadirachtin) and Allium sativum (allicin) and their binary combinations have sufficient larvicidal activity against different larva of $F$. gigantica in in vitro treatments. The alcoholic extract of bulbs of $A$. sativum has also shown moderate in vitro antihelminitic activity against human Ascaris lumbricoides ${ }^{18}$. A. sativum has been reported to be effective in dysentery and also act as vermifuge ${ }^{17,21}$. Oil of A. sativum has also been reported to possess antihelmintic activity ${ }^{8,10,29}$ and discards all injurious parasites in the intestine ${ }^{17}$. In vitro and in vivo efficacy of single treatments of the active components citral, ferulic acid, umbelliferone, azadirachtin and allicin against redia and cercaria larva of $F$. gigantica have potent larvicidal activity ${ }^{30}$.

Z. officinale is a perennial plant and is considered to be the universal medicine in Ayurveda. The antihelminthic activity of ethanolic extracts of rhizomes of Z. officinale against human Ascaris lumbricoides is appreciable ${ }^{18}$. GOTO et al. ${ }^{7}$ reported the lethal effect of $Z$. officinale on Anisakis larvae in vitro. The anti-filarial effect of $Z$. officinale against Dirofilaria immitis has been reported by DATTA \& SUKUL ${ }^{4}$. ADEWUNMI et al. ${ }^{1}$, SINGH et al. ${ }^{26}$ have reported the molluscicidal activity of $Z$. officinale.

Ferulic acid and umbelliferone extracted from Ferula asafoetida root latex are potent molluscicides against $L$. acuminata $^{11,12,13}$. The antioxidant, anticarcinogenic, antispasmodic, antihelminthic activity of $F$. asafoetida extract and ferulic acid were reported by various workers ${ }^{5,6,20}$. Spigelia anthelmia inhibits the motility of Haemonchus contortus larva ${ }^{3}$, SINGH et $a l .{ }^{24}$ observed that $A$. indica have molluscicidal activity against $L$. acuminata. In vitro treatment of citral, ferulic acid, umbelliferone, azadirachtin and allicin caused toxicity against redia $\left(8 \mathrm{hCC}_{50} 4.14,0.45\right.$, $0.63,0.07$ and $0.01 \mathrm{mg} / \mathrm{L}$, respectively) and cercaria $\left(8 \mathrm{~h} \mathrm{EC}_{50} 6.08,0.44\right.$, $0.27,0.08$ and $0.009 \mathrm{mg} / \mathrm{L}$, respectively) larva of $F$. gigantica ${ }^{30}$. Binary combinations of ferulic acid + azadirachtin against redia larva is 64.28 times more effective than single treatment of ferulic acid, where as in case of cercaria citral + allicin was 53.80 times more effective than single treatment of citral (Table 3). Earlier it was reported that citral $(24 \mathrm{~h} \mathrm{EC}$ - $68.95 \mathrm{mg} / \mathrm{L})$, ferulic acid $\left(24 \mathrm{~h} \mathrm{EC}_{50}-2.21 \mathrm{mg} / \mathrm{L}\right)$, umbelliferone $(24 \mathrm{~h}$ $\left.\mathrm{EC}_{50}-3.43 \mathrm{mg} / \mathrm{L}\right)$, azadirachtin $\left(24 \mathrm{~h} \mathrm{EC} \mathrm{E}_{50}-0.35 \mathrm{mg} / \mathrm{L}\right)$, and allicin $(24 \mathrm{~h}$ $\mathrm{EC}_{50}-6.34 \mathrm{mg} / \mathrm{L}$ ) are potent molluscicides against $L$. acuminata ${ }^{11,23,26,27}$. $8 \mathrm{~h} \mathrm{EC}{ }_{50}$ of binary combinations of these larvicidal components against redia/cercaria larva is 1.25 (allicin + umbelliferone)/ 1.23 (citral + umbelliferone) times low that kill the intermediate host L. acuminata. Different concentrations of binary combinations used to kill redia and cercaria are not toxic to the snail, even in a $24 \mathrm{~h}$ exposure period. Use of these plants and their active component in killing the redia and cercaria of $F$. gigantica without killing the host snail is important. The snail is a crucial component of aquatic ecosystem. The present studies clearly demonstrate that direct treatment of the redia and cercaria will be a more efficient method to control fascioliasis.

The steep slope value indicates that a small increase in the concentration of different larvicide caused higher larval mortality. A t-ratio value greater than 1.96 indicates that the regression is significant. The heterogeneity factor value of less than 1.0 denotes that in the replicate test of random samples, the concentration response is limited and, thus, the model fits the data adequately. The index of significance of the potency estimation $g$ indicates that the value of the mean is within the limit at all probability levels $\left(90,95\right.$, and 99 , respectively) since it is less than $0.5^{19}$.

It can be concluded from the present study that binary combination of these natural products will be more helpful in controlling the redia/ cercaria than their single components, because their larvicidal effect is 1.25 to $64.28 / 1.23$ to 53.80 more effective in killing the larva. The effective toxic concentration in the binary combinations of each larvicidal component is lower and would be safer in aquatic environment.

\section{RESUMO}

\section{Fitoterapia in vitro de caramujos vetores por combinações binárias de componentes ativos larvicidas como controle efetivo de fasciolíase}

A infecção alimentar pelo trematóide da fasciolíase é uma dentre os mais comuns problemas de saúde pública mundiais, causando grande prejuízo econômico para a humanidade. Controle da população de caramujos abaixo de determinado nível é um dos métodos no campo mais importantes para a redução da incidência da fasciolíase. O ciclo de vida do parasita pode ser interrompido pela morte do caramujo ou da larva redia e cercária da Fasciola dentro da Lymnaea acuminata. Foi testada a toxicidade in vitro das diferentes combinações binárias (relação 1:1) entre os vários componentes larvicidas ativos da planta tais como citral, 


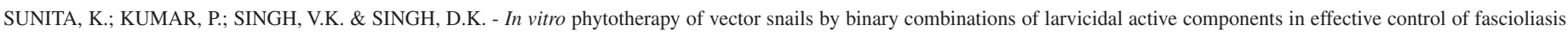
Rev. Inst. Med. Trop. Sao Paulo, 55(5): 303-8, 2013.

Table 1

In vitro toxicity of different binary combinations (1:1 ratio) of active larvicidal components against the redia larva of $F$. gigantica

\begin{tabular}{|c|c|c|c|c|c|c|c|c|}
\hline $\begin{array}{l}\text { Exposure } \\
\text { period }\end{array}$ & Treatment & $\begin{array}{c}\mathrm{EC}_{50} \mathrm{mg} / \mathrm{L} \\
(\mathrm{w} / \mathrm{v})\end{array}$ & Limits LCL & Limits UCL & Slope value & t-ratio & g-value & Heterogeneity \\
\hline \multirow[t]{10}{*}{$2 \mathrm{~h}$} & $\mathrm{Ci}+\mathrm{Fe}$ & 29.500 & 17.18 & 13.84 & $1.050 \pm 0.306$ & 3.439 & 0.325 & 0.13 \\
\hline & $\mathrm{Ci}+\mathrm{Um}$ & 18.532 & 12.28 & 51.62 & $1.709 \pm 0.408$ & 4.190 & 0.219 & 0.29 \\
\hline & $\mathrm{Ci}+\mathrm{Az}$ & 5.700 & 3.806 & 21.58 & $1.338 \pm 0.401$ & 3.334 & 0.346 & 0.15 \\
\hline & $\mathrm{Ci}+\mathrm{Al}$ & 0.547 & 0.364 & 2.246 & $1.266 \pm 0.394$ & 3.210 & 0.373 & 0.11 \\
\hline & $\mathrm{Fe}+\mathrm{Um}$ & 2.401 & 1.573 & 10.07 & $1.350 \pm 0.407$ & 3.313 & 0.350 & 0.14 \\
\hline & $\mathrm{Fe}+\mathrm{Az}$ & 0.041 & 0.030 & 0.081 & $1.091 \pm 0.330$ & 3.302 & 0.390 & 0.16 \\
\hline & $\mathrm{Fe}+\mathrm{Al}$ & 0.062 & 0.044 & 0.096 & $1.284 \pm 0.429$ & 2.995 & 0.428 & 0.09 \\
\hline & $\mathrm{Az}+\mathrm{Um}$ & 0.433 & 0.358 & 0.659 & $1.986 \pm 0.568$ & 3.493 & 0.315 & 0.13 \\
\hline & $\mathrm{Az}+\mathrm{Al}$ & 0.015 & 0.010 & 0.021 & $1.308 \pm 0.382$ & 3.420 & 0.382 & 0.15 \\
\hline & $\mathrm{Al}+\mathrm{Um}$ & 0.040 & 0.027 & 0.152 & $1.073 \pm 0.374$ & 2.869 & 0.466 & 0.10 \\
\hline \multirow[t]{10}{*}{$4 \mathrm{~h}$} & $\mathrm{Ci}+\mathrm{Fe}$ & 15.330 & 10.31 & 40.30 & $0.998 \pm 0.286$ & 3.491 & 0.315 & 0.13 \\
\hline & $\mathrm{Ci}+\mathrm{Um}$ & 15.704 & 9.762 & 68.67 & $1.140 \pm 0.330$ & 3.454 & 0.322 & 0.17 \\
\hline & $\mathrm{Ci}+\mathrm{Az}$ & 3.720 & 2.662 & 9.86 & $1.167 \pm 0.375$ & 3.115 & 0.396 & 0.13 \\
\hline & $\mathrm{Ci}+\mathrm{Al}$ & 0.346 & 0.247 & 0.892 & $1.124 \pm 0.372$ & 3.023 & 0.420 & 0.10 \\
\hline & $\mathrm{Fe}+\mathrm{Um}$ & 1.553 & 1.111 & 4.450 & $1.214 \pm 0.392$ & 3.094 & 0.401 & 0.11 \\
\hline & $\mathrm{Fe}+\mathrm{Az}$ & 0.024 & 0.016 & 0.038 & $1.046 \pm 0.324$ & 3.230 & 0.368 & 0.13 \\
\hline & $\mathrm{Fe}+\mathrm{Al}$ & 0.039 & 0.019 & 0.050 & $1.413 \pm 0.433$ & 3.267 & 0.360 & 0.12 \\
\hline & $\mathrm{Az}+\mathrm{Um}$ & 0.319 & 0.247 & 0.397 & $1.916 \pm 0.557$ & 3.437 & 0.325 & 0.13 \\
\hline & $\mathrm{Az}+\mathrm{Al}$ & 0.010 & 0.005 & 0.013 & $1.370 \pm 0.391$ & 3.501 & 0.313 & 0.15 \\
\hline & $\mathrm{Al}+\mathrm{Um}$ & 0.022 & 0.014 & 0.033 & $1.129 \pm 0.365$ & 3.091 & 0.402 & 0.13 \\
\hline \multirow[t]{10}{*}{$6 \mathrm{~h}$} & $\mathrm{Ci}+\mathrm{Fe}$ & 8.107 & 5.239 & 12.78 & $0.996 \pm 0.280$ & 3.561 & 0.303 & 0.14 \\
\hline & $\mathrm{Ci}+\mathrm{Um}$ & 9.500 & 6.392 & 28.46 & $0.995 \pm 0.309$ & 3.223 & 0.370 & 0.15 \\
\hline & $\mathrm{Ci}+\mathrm{Az}$ & 2.218 & 1.435 & 3.41 & $1.112 \pm 0.365$ & 3.045 & 0.414 & 0.13 \\
\hline & $\mathrm{Ci}+\mathrm{Al}$ & 0.202 & 0.119 & 0.301 & $1.084 \pm 0.364$ & 2.975 & 0.434 & 0.10 \\
\hline & $\mathrm{Fe}+\mathrm{Um}$ & 0.987 & 0.687 & 1.66 & $1.156 \pm 0.390$ & 2.965 & 0.437 & 0.11 \\
\hline & $\mathrm{Fe}+\mathrm{Az}$ & 0.014 & 0.005 & 0.020 & $1.076 \pm 0.327$ & 3.290 & 0.355 & 0.14 \\
\hline & $\mathrm{Fe}+\mathrm{Al}$ & 0.026 & 0.008 & 0.038 & $1.410 \pm 0.446$ & 3.174 & 0.381 & 0.20 \\
\hline & $\mathrm{Az}+\mathrm{Um}$ & 0.234 & 0.143 & 0.287 & $1.985 \pm 0.569$ & 3.437 & 0.310 & 0.15 \\
\hline & $\mathrm{Az}+\mathrm{Al}$ & 0.007 & 0.003 & 0.009 & $1.566 \pm 0.418$ & 3.750 & 0.273 & 0.18 \\
\hline & $\mathrm{Al}+\mathrm{Um}$ & 0.013 & 0.005 & 0.018 & $1.157 \pm 0.367$ & 3.152 & 0.387 & 0.15 \\
\hline \multirow[t]{10}{*}{$8 \mathrm{~h}$} & $\mathrm{Ci}+\mathrm{Fe}$ & 4.463 & 1.968 & 6.50 & $1.023 \pm 0.282$ & 3.624 & 0.292 & 0.15 \\
\hline & $\mathrm{Ci}+\mathrm{Um}$ & 5.172 & 3.187 & 8.63 & $0.948 \pm 0.301$ & 3.148 & 0.387 & 0.15 \\
\hline & $\mathrm{Ci}+\mathrm{Az}$ & 1.304 & 0.470 & 1.84 & $1.136 \pm 0.366$ & 3.100 & 0.400 & 0.14 \\
\hline & $\mathrm{Ci}+\mathrm{Al}$ & 0.118 & 0.035 & 0.171 & $1.119 \pm 0.367$ & 3.048 & 0.413 & 0.11 \\
\hline & $\mathrm{Fe}+\mathrm{Um}$ & 0.607 & 0.256 & 0.831 & $1.186 \pm 0.398$ & 2.977 & 0.433 & 0.14 \\
\hline & $\mathrm{Fe}+\mathrm{Az}$ & 0.007 & 0.002 & 0.013 & $1.145 \pm 0.340$ & 3.367 & 0.378 & 0.28 \\
\hline & $\mathrm{Fe}+\mathrm{Al}$ & 0.022 & 0.001 & 0.012 & $1.092 \pm 0.342$ & 3.342 & 0.243 & 0.29 \\
\hline & $\mathrm{Az}+\mathrm{Um}$ & 0.183 & 0.103 & 0.230 & $2.366 \pm 0.597$ & 3.966 & 0.244 & 0.29 \\
\hline & $\mathrm{Az}+\mathrm{Al}$ & 0.006 & 0.003 & 0.008 & $2.324 \pm 0.516$ & 4.401 & 0.190 & 0.33 \\
\hline & $\mathrm{Al}+\mathrm{Um}$ & 0.008 & 0.002 & 0.013 & $1.355 \pm 0.355$ & 3.537 & 0.307 & 0.27 \\
\hline
\end{tabular}

$\mathrm{Ci}=$ citral, $\mathrm{Fe}=$ ferulic acid, $\mathrm{Um}=$ umbelliferone, $\mathrm{Az}=$ azadirachtin, $\mathrm{Al}=$ allicin, $\mathrm{LCL}-$ lower confidence limits, UCL - upper confidence limits. Six batches of ten redia larva were exposed to different concentration of binary combinations of the above larvicides treatments. Mortality was recorded every $2 \mathrm{~h}$. Concentrations given are the final concentration (W/V) in the glass aquarium water. Significant negative regression $(p<0.05)$ was observed between exposure time and $\mathrm{EC}_{50}$ of treatments. Ts - testing significant of the regression coefficient. $\mathrm{Ci}+\mathrm{Fe}:-7.76^{++}, \mathrm{Ci}+\mathrm{Um}:-10.50^{+}, \mathrm{Ci}+\mathrm{Az}:-8.70^{+}, \mathrm{Ci}+\mathrm{Al}:-7.73^{+}, \mathrm{Fe}+\mathrm{Um}:-8.00^{+}, \mathrm{Fe}+\mathrm{Az}:-6.97^{+}, \mathrm{Fe}+\mathrm{Al}:-12.34^{+}$, Az+Um:-8.67++ Az+Al:-16.24++, Al+Um:-1.59+. +: linear regression between $x$ and $y$; ++: non - linear regression between log $x$ and log $y$. 
SUNITA, K.; KUMAR, P.; SINGH, V.K. \& SINGH, D.K. - In vitro phytotherapy of vector snails by binary combinations of larvicidal active components in effective control of fascioliasis. Rev. Inst. Med. Trop. Sao Paulo, 55(5): 303-8, 2013.

Table 2

In vitro toxicity of different binary combination of active larvicidal components against the cercaria larva of $F$. gigantica.

\begin{tabular}{|c|c|c|c|c|c|c|c|c|}
\hline $\begin{array}{l}\text { Exposure } \\
\text { period }\end{array}$ & Treatment & $\mathrm{EC}_{50} \mathrm{mg} / \mathrm{l}(\mathrm{w} / \mathrm{v})$ & Limits LCL & Limits UCL & Slope value & t-ratio & g-value & Heterogeneity \\
\hline \multirow[t]{10}{*}{$2 \mathrm{~h}$} & $\mathrm{Ci}+\mathrm{Fe}$ & 34.962 & 20.928 & 140.729 & $1.330 \pm 0.340$ & 3.917 & 0.250 & 0.18 \\
\hline & $\mathrm{Ci}+\mathrm{Um}$ & 19.579 & 12.392 & 67.064 & $1.525 \pm 0.386$ & 3.947 & 0.246 & 0.23 \\
\hline & $\mathrm{Ci}+\mathrm{Az}$ & 5.364 & 3.618 & 19.816 & $1.297 \pm 0.395$ & 3.284 & 0.356 & 0.12 \\
\hline & $\mathrm{Ci}+\mathrm{Al}$ & 0.567 & 0.369 & 2.851 & $1.213 \pm 0.392$ & 3.096 & 0.401 & 0.12 \\
\hline & $\mathrm{Fe}+\mathrm{Um}$ & 2.457 & 1.608 & 10.004 & $1.379 \pm 0.409$ & 3.370 & 0.338 & 0.11 \\
\hline & $\mathrm{Fe}+\mathrm{Az}$ & 0.044 & 0.031 & 0.109 & $1.165 \pm 0.334$ & 3.486 & 0.316 & 0.10 \\
\hline & $\mathrm{Fe}+\mathrm{Al}$ & 0.079 & 0.062 & 0.129 & $1.575 \pm 0.438$ & 3.592 & 0.298 & 0.11 \\
\hline & $\mathrm{Az}+\mathrm{Um}$ & 0.441 & 0.371 & 0.627 & $2.247 \pm 0.577$ & 3.892 & 0.253 & 0.13 \\
\hline & $\mathrm{Az}+\mathrm{Al}$ & 0.018 & 0.012 & 0.032 & $1.115 \pm 0.380$ & 2.933 & 0.446 & 0.10 \\
\hline & $\mathrm{Al}+\mathrm{Um}$ & 0.035 & 0.027 & 0.065 & $1.398 \pm 0.381$ & 3.671 & 0.285 & 0.13 \\
\hline \multirow[t]{10}{*}{$4 \mathrm{~h}$} & $\mathrm{Ci}+\mathrm{Fe}$ & 24.654 & 15.072 & 107.980 & $1.036 \pm 0.298$ & 3.477 & 0.318 & 0.13 \\
\hline & $\mathrm{Ci}+\mathrm{Um}$ & 10.942 & 7.931 & 21.679 & $1.415 \pm 0.330$ & 4.290 & 0.209 & 0.27 \\
\hline & $\mathrm{Ci}+\mathrm{Az}$ & 3.577 & 2.529 & 10.369 & $1.098 \pm 0.372$ & 2.951 & 0.441 & 0.10 \\
\hline & $\mathrm{Ci}+\mathrm{Al}$ & 0.352 & 0.248 & 1.026 & $1.082 \pm 0.371$ & 2.919 & 0.451 & 0.12 \\
\hline & $\mathrm{Fe}+\mathrm{Um}$ & 1.713 & 1.181 & 6.953 & $1.148 \pm 0.392$ & 2.925 & 0.449 & 0.09 \\
\hline & $\mathrm{Fe}+\mathrm{Az}$ & 0.028 & 0.019 & 0.050 & $1.028 \pm 0.324$ & 3.168 & 0.383 & 0.15 \\
\hline & $\mathrm{Fe}+\mathrm{Al}$ & 0.054 & 0.038 & 0.072 & $1.454 \pm 0.430$ & 3.379 & 0.336 & 0.11 \\
\hline & $\mathrm{Az}+\mathrm{Um}$ & 0.355 & 0.283 & 0.477 & $1.826 \pm 0.558$ & 3.274 & 0.358 & 0.13 \\
\hline & $\mathrm{Az}+\mathrm{Al}$ & 0.011 & 0.005 & 0.014 & $1.213 \pm 0.386$ & 3.143 & 0.389 & 0.16 \\
\hline & $\mathrm{Al}+\mathrm{Um}$ & 0.022 & 0.015 & 0.033 & $1.178 \pm 0.366$ & 3.216 & 0.371 & 0.11 \\
\hline \multirow[t]{10}{*}{$6 \mathrm{~h}$} & $\mathrm{Ci}+\mathrm{Fe}$ & 13.986 & 9.277 & 38.423 & $0.926 \pm 0.283$ & 3.276 & 0.358 & 0.12 \\
\hline & $\mathrm{Ci}+\mathrm{Um}$ & 8.974 & 6.111 & 24.322 & $1.005 \pm 0.308$ & 3.262 & 0.361 & 0.14 \\
\hline & $\mathrm{Ci}+\mathrm{Az}$ & 2.067 & 1.204 & 3.157 & $1.056 \pm 0.364$ & 2.899 & 0.457 & 0.10 \\
\hline & $\mathrm{Ci}+\mathrm{Al}$ & 0.202 & 0.113 & 0.306 & $1.046 \pm 0.364$ & 2.875 & 0.464 & 0.12 \\
\hline & $\mathrm{Fe}+\mathrm{Um}$ & 0.939 & 0.688 & 1.454 & $1.232 \pm 0.371$ & 3.059 & 0.346 & 0.09 \\
\hline & $\mathrm{Fe}+\mathrm{Az}$ & 0.016 & 0.007 & 0.022 & $1.037 \pm 0.325$ & 3.192 & 0.377 & 0.16 \\
\hline & $\mathrm{Fe}+\mathrm{Al}$ & 0.036 & 0.019 & 0.047 & $1.510 \pm 0.436$ & 3.462 & 0.320 & 0.13 \\
\hline & $\mathrm{Az}+\mathrm{Um}$ & 0.255 & 0.167 & 0.310 & $1.939 \pm 0.559$ & 3.467 & 0.320 & 0.12 \\
\hline & $\mathrm{Az}+\mathrm{Al}$ & 0.008 & 0.123 & 0.320 & $1.950 \pm 0.651$ & 3.321 & 0.421 & 0.18 \\
\hline & $\mathrm{Al}+\mathrm{Um}$ & 0.015 & 0.007 & 0.020 & $1.219 \pm 0.367$ & 3.325 & 0.347 & 0.17 \\
\hline \multirow[t]{10}{*}{$8 \mathrm{~h}$} & $\mathrm{Ci}+\mathrm{Fe}$ & 7.092 & 4.030 & 11.147 & $0.920 \pm 0.278$ & 3.304 & 0.352 & 0.11 \\
\hline & $\mathrm{Ci}+\mathrm{Um}$ & 4.911 & 2.969 & 7.862 & $0.963 \pm 0.301$ & 3.196 & 0.376 & 0.14 \\
\hline & $\mathrm{Ci}+\mathrm{Az}$ & 1.189 & 0.323 & 1.729 & $1.088 \pm 0.367$ & 2.966 & 0.436 & 0.11 \\
\hline & $\mathrm{Ci}+\mathrm{Al}$ & 0.113 & 0.027 & 0.166 & $1.076 \pm 0.367$ & 2.920 & 0.448 & 0.12 \\
\hline & $\mathrm{Fe}+\mathrm{Um}$ & 0.595 & 0.273 & 0.803 & $1.257 \pm 0.400$ & 3.143 & 0.389 & 0.10 \\
\hline & $\mathrm{Fe}+\mathrm{Az}$ & 0.009 & 0.002 & 0.014 & $1.128 \pm 0.336$ & 3.356 & 0.341 & 0.19 \\
\hline & $\mathrm{Fe}+\mathrm{Al}$ & 0.026 & 0.011 & 0.036 & $1.736 \pm 0.461$ & 3.765 & 0.271 & 0.48 \\
\hline & $\mathrm{Az}+\mathrm{Um}$ & 0.193 & 0.096 & 0.246 & $2.023 \pm 0.575$ & 3.516 & 0.311 & 0.12 \\
\hline & $\mathrm{Az}+\mathrm{Al}$ & 0.005 & 0.002 & 0.008 & $1.589 \pm 0.454$ & 3.718 & 0.278 & 0.29 \\
\hline & $\mathrm{Al}+\mathrm{Um}$ & 0.010 & 0.004 & 0.014 & $1.468 \pm 0.381$ & 3.848 & 0.259 & 0.32 \\
\hline
\end{tabular}

$\mathrm{Ci}=$ citral, $\mathrm{Fe}=$ ferulic acid, $\mathrm{Um}=$ umbelliferone, $\mathrm{Az}=$ azadirachtin, $\mathrm{Al}=$ allicin, $\mathrm{LCL}$ - lower confidence limits, UCL - upper confidence limits. Six batches of ten cercaria larva were exposed to different concentration of binary combinations of the above larvicides treatments. Mortality was recorded every $2 \mathrm{~h}$. Concentrations given are the final concentration $(\mathrm{W} / \mathrm{V})$ in the glass aquarium water. Significant negative regression $(p<0.05)$ was observed between exposure time and $\mathrm{EC}_{50}$ of treatments. Ts - testing significant of the regression coefficient. $\mathrm{Ci}+\mathrm{Fe}:-15.33^{+}, \mathrm{Ci}+\mathrm{Um}:-5.53^{++}, \mathrm{Ci}+\mathrm{Az}:-9.61^{+}, \mathrm{Ci}+\mathrm{Al}:-7.58^{+}, \mathrm{Fe}+\mathrm{Um}:-8.94^{+}, \mathrm{Fe}+\mathrm{Az}:-8.21^{+}, \mathrm{Fe}+\mathrm{Al}:-7.97^{++}$, $\mathrm{Az}+\mathrm{Um}:-15.97^{+}, \mathrm{Az}+\mathrm{Al}:-7.41^{+}, \mathrm{Al}+\mathrm{Um}:-8.51^{++} .+$: linear regression between $x$ and $y$; ++: non - linear regression between $\log x$ and $\log y$. 


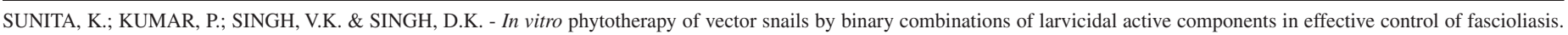
Rev. Inst. Med. Trop. Sao Paulo, 55(5): 303-8, 2013.

Table 3

Synergism in the in vitro larvicidal activity of different binary combinations against redia and cercaria larva of $F$. gigantica at 8 h exposure period

\begin{tabular}{|c|c|c|c|c|}
\hline \multirow[b]{2}{*}{ Treatment } & \multicolumn{2}{|c|}{ Redia } & \multicolumn{2}{|c|}{ Cercaria } \\
\hline & $8 \mathrm{~h} \mathrm{EC}_{50} \mathrm{mg} / \mathrm{L}$ & $\begin{array}{c}\text { Synergistic } \\
\text { ratio }\end{array}$ & $8 \mathrm{~h} \mathrm{EC}_{50} \mathrm{mg} / \mathrm{L}$ & $\begin{array}{c}\text { Synergistic } \\
\text { ratio }\end{array}$ \\
\hline$\overline{\mathrm{Ci}}$ & 4.14 & - & 6.08 & - \\
\hline $\mathrm{Fe}$ & 0.45 & - & 0.44 & - \\
\hline $\mathrm{Um}$ & 0.63 & - & 0.27 & - \\
\hline $\mathrm{Az}$ & 0.07 & - & 0.08 & - \\
\hline $\mathrm{Al}$ & 0.01 & - & 0.009 & - \\
\hline $\mathrm{Ci}+\mathrm{Fe}$ & 4.463 & - & 7.092 & - \\
\hline $\mathrm{Ci}+\mathrm{Um}$ & 5.172 & - & 4.911 & 1.23 \\
\hline $\mathrm{Ci}+\mathrm{Az}$ & 1.304 & 3.18 & 1.189 & 5.15 \\
\hline $\mathrm{Ci}+\mathrm{Al}$ & 0.118 & 37.37 & 0.113 & 53.80 \\
\hline $\mathrm{Fe}+\mathrm{Um}$ & 0.607 & - & 0.595 & - \\
\hline $\mathrm{Fe}+\mathrm{Az}$ & 0.007 & 64.28 & 0.009 & 48.88 \\
\hline $\mathrm{Fe}+\mathrm{Al}$ & 0.022 & 20.45 & 0.026 & 16.92 \\
\hline $\mathrm{Az}+\mathrm{Um}$ & 0.183 & - & 0.193 & - \\
\hline $\mathrm{Az}+\mathrm{Al}$ & 0.006 & 11.66 & 0.005 & 16.00 \\
\hline $\mathrm{Al}+\mathrm{Um}$ & 0.008 & 1.25 & 0.010 & - \\
\hline
\end{tabular}

$\mathrm{Ci}=$ Citral, $\mathrm{Fe}=$ Ferulic acid, $\mathrm{Um}=$ Umbelliferone, $\mathrm{Az}=$ Azadirachtin, $\mathrm{Al}=$ Allicin. Single treatments of active larvicides were reported by SUNITA and SINGH (2011).

ácido ferúlico, umbeliferone, azadiractina, e alicina contra a Fasciola redia e a cercária. A mortalidade das larvas foi observada após duas, quatro, seis e oito horas de tratamento. A condição in vitro azadiractina + alicina (relação 1:1) foi altamente tóxica contra redia e cercária ( $8 \mathrm{~h}$ $\mathrm{LC}_{50} 0,006$ e $0,005 \mathrm{mg} / \mathrm{L}$ ). Toxicidade do citral + ácido ferúlico foi a mais baixa contra redia e larvas de cercária.

\section{ACKNOWLEDGMENTS}

One of the authors (Kumari Sunita) is thankful to Rajiv Gandhi National Fellowship (RGNF), University Grants Commission (UGC), New Delhi for financial assistance.

\section{REFERENCES}

1. Adewunmi CO, Oguntimein BO, Furu P. Molluscicidal and antischistosomal activities of Zingiber officinale. Planta Med. 1990;56:374-6.

2. Agarwal RA, Singh DK. Harmful gastropods and their control. Acta Hydrochim Hydrobiol. 1988;16:113-38.

3. Assis LM, Bevilequa CML, Morais SM, Vieira LS, Costa CTC, Souza JAL. Ovicidal and larvicidal activity in vitro of Spigelia anthelmia Linn. extract on Haemonchus contortus. Vet Parasitol. 2003;117:43-9.

4. Datta A, Sukul NC. Antifilarial effect of Zingiber officinale on Dirofilaria immitis. J Helminthol. 1987;61:268-70.
5. Eigner D, Scholz D. Ferula asafoetida and Curcuma longa in traditional treatment and diet in Nepal. J Ethnopharmacol. 1999;67:1-6.

6. Fatehi M, Farifteh F, Fatehi-Hassanabad Z. Antispasmodic and hypotensive effects of Ferula asafoetida gum extract. J Ethnopharmacol. 2004;91:321-4.

7. Goto C, Kasuya S, Koga K, Ohtomo H, Kaget N. Lethal efficacy of extract from Zingiber officinale (traditional Chinese medicine) or [6]-shogaol and [6]-gingerol in Anisakis larvae in vitro. Parasitol Res. 1990;76:653-6.

8. Hoppe HA, Drogenkunde. Angiosperms. $8^{\text {th }}$ ed. Berlin: Walter De Gruyter; 1975. v.

9. Jaiswal P, Singh DK. Molluscicidal activity of Nutmeg and Mace (Myristica fragrans Houtt.) against the vector snail Lymnaea acuminata. J Herbs Spices Medicinal Plants. $2009 ; 15: 177-86$

10. Kirtikar KR, Basu BD. Indian medicinal plants. Part II. Bahadurgay: Indian Press 1981

11. Kumar P, Singh DK. Molluscicidal activity of Ferula asafoetida, Syzygium aromaticum and Carum carvi and their active components against the snail Lymnaea acuminata. Chemosphere. 2006;63:1568-74.

12. Kumar P, Singh VK, Singh DK. Bait formulations of molluscicides and their effects on biochemical changes in the ovotestis of snail Lymnaea acuminata (Mollusca Gastropoda: Lymnaeidae). Rev Inst Med Trop Sao Paulo. 2011;53:271-5.

13. Kumar P, Singh VK, Singh DK. Kinetics of enzyme inhibition by active molluscicidal agent ferulic acid, umbelliferone, eugenol and limonene in the nervous tissues of snai Lymnaea acuminata. Phytother Res. 2009;23:172-7.

14. Mas-Coma S, Bargues MD, Valero MA. Fascioliasis and other plant borne trematode zoonoses. Int J Parasitol. 2005;35:1255-78.

15. Mas-Coma S, Estebam JG, Bargues MD. Epidemiology of human fascioliasis: a review and proposed new classification. Bull World Health Organ. 1999;77:340-6.

16. Mas-Coma S, Valero MA, Bargues MD. Chapter 2: Fasciola Lymnaeids an human fascioliasis with a global overview on disease transmission, epidemiology, evolutionary genetics, molecular epidemiology and control. Adv Parasitol 2009;69:41-146

17. Nadkarni KM. Indian Materia Medica. Popular Prakashan. Bombay: Private Limited 1976. v. 1 and 2 .

18. Raj KR. Screening of indigenous plant for anthelmintic action against human Ascaris lumbricoides: part II. Indian J Physiol Pharmacol. 1975;19:47-50.

19. Robertson JL, Russell RM, Preciter HK, Savin NE. Bioassay with arthropods data $2^{\text {nd }}$ ed. Boca Raton: Taylor and Francis, CRC Press; 2007. p. 1-224.

20. Saleem M, Alam A, Sultana S. Asafoetida inhibits early events of carcinogenesis: a chemopreventive study. Life Sci. 2001;68:1913-21.

21. Schavenberg P, Paris F. Guide to medicinal plants. London: Lutterworth Press; 1977

22. Singh V, Singh DK. The effect of abiotic factor on the toxicity of cypermethrin against the snail Lymnaea acuminata in the control of fascioliasis. J Helminthol. 2009;83:39-45

23. Singh K, Singh A, Singh DK. Molluscicidal activity of different combinations of the plant products used in the molluscicides Pestoban. Biol Agric Hortic. 1995;12:253-61.

24. Singh K, Singh A, Singh DK. Molluscicidal activity of neem (Azadirachta indica A Juss). J Ethnopharmacol. 1996;52:35-40.

25. Singh O, Agarwal RA. Toxicity of certain pesticides to two economic species of snail in northern India. J Econ Entomol. 1981;74:568-71. 
SUNITA, K.; KUMAR, P.; SINGH, V.K. \& SINGH, D.K. - In vitro phytotherapy of vector snails by binary combinations of larvicidal active components in effective control of fascioliasis Rev. Inst. Med. Trop. Sao Paulo, 55(5): 303-8, 2013.

26. Singh S, Singh VK, Singh DK. Molluscicidal activity of some common spice plants. Biol Agric Hortic. 1997;14:237-49.

27. Singh VK, Singh DK. Characterization of allicin as a molluscicidal agent in Allium sativum (garlic). Biol Agric Hortic. 1995;12:119-31.

28. Sokal RR, Rohlf FJ. Introduction of biostatistics. San Francisco: W.H. Freeman; 1996.
29. Steenis-Kruseman MJV. Select Indonesian medicinal plants organize. Sci Res Indonesia Bull. 1953;18:31.

30. Sunita K, Singh DK. Fascioliasis control: in vivo and in vitro phytotherapy of vector snail to kill Fasciola larva. J Parasitol Res. 2011;2011:1-7.

Received: 1 April 2012

Accepted: 13 December 2012 